\title{
Synergistic stimulation of type I interferons during influenza virus coinfection promotes Streptococcus pneumoniae colonization in mice
}

\author{
Shigeki Nakamura, ${ }^{1,2}$ Kimberly M. Davis, ${ }^{1}$ and Jeffrey N. Weiser ${ }^{1}$ \\ 1Department of Microbiology, University of Pennsylvania, Philadelphia, Pennsylvania, USA. ${ }^{2}$ Department of Molecular Microbiology and Immunology, \\ Nagasaki University Graduate School of Biomedical Sciences, Nagasaki, Japan.
}

\begin{abstract}
Pneumococcal infection of the respiratory tract is often secondary to recent influenza virus infection and accounts for much of the morbidity and mortality during seasonal and pandemic influenza. Here, we show that coinfection of the upper respiratory tract of mice with influenza virus and pneumococcus leads to synergistic stimulation of type I IFNs and that this impairs the recruitment of macrophages, which are required for pneumococcal clearance, due to decreased production of the chemokine CCL2. Type I IFN expression was induced by pneumococcal colonization alone. Colonization followed by influenza coinfection led to a synergistic type I IFN response, resulting in increased density of colonizing bacteria and susceptibility to invasive infection. This enhanced type I IFN response inhibited production of the chemokine CCL2, which promotes the recruitment of macrophages and bacterial clearance. Stimulation of CCL2 by macrophages upon pneumococcal infection alone required the pattern recognition receptor Nod 2 and expression of the pore-forming toxin pneumolysin. Indeed, the increased colonization associated with concurrent influenza virus infection was not observed in mice lacking Nod2 or the type I IFN receptor, or in mice challenged with pneumococci lacking pneumolysin. We therefore propose that the synergistic stimulation of type I IFN production during concurrent influenza virus and pneumococcal infection leads to increased bacterial colonization and suggest that this may contribute to the higher rates of disease associated with coinfection in humans.
\end{abstract}

\section{Introduction}

Streptococcus pneumoniae (the pneumococcus) transiently colonizes the mucosal surfaces of the human upper respiratory tract (URT) (1). Colonizing organisms in the nasopharynx provide the reservoir for transmission of the pneumococcus within the population. Under certain conditions, the organism may overwhelm the mucosal defenses of the URT and transit to normally sterile parts of the upper or lower airway or beyond. Because colonization and factors that allow the spread of organisms outside their niche in the URT are common, the pneumococcus is a leading cause of bacterial diseases, including otitis media, pneumonia, and sepsis. A recent survey of respiratory tract infection in children demonstrated that viral infection is temporally associated with an approximately 15 -fold increase in the number of pneumococci detected in nasal cultures (2). Moreover, viral infection of the respiratory tract is frequently associated with increased incidence of invasive pneumococcal diseases (3-5). Influenza virus, in particular, predisposes to secondary pneumococcal pneumonia, and this coinfection accounts for much of the increased morbidity and mortality during seasonal and pandemic influenza (4).

A number of reports have used a murine model of coinfection to examine the mechanisms responsible for the higher incidence of pneumococcal infection of the lower respiratory tract (LRT) following influenza (secondary pneumococcal pneumonia). An impairment of macrophage or neutrophil function, upregulation

Conflict of interest: The authors have declared that no conflict of interest exists. Citation for this article: J Clin Invest. 2011;121(9):3657-3665. doi:10.1172/JCI57762. of the platelet-activating factor receptor, and induction of the antiinflammatory cytokine IL-10 have each been implicated in promoting post-influenza secondary pneumococcal pneumonia (6-8). Although during natural influenza virus infection the mucosal surfaces of the URT are a prominent site of viral replication, these studies have relied on direct inoculation of bacteria and virus into the LRT and have not examined the effect of influenza infection on pneumococcal colonization localized to the URT (9).

In considering how viral infection impacts pneumococcal colonization, we focused on the role of IFNs, particularly type I IFNs, since we had previously determined that colonization is not affected in mice lacking the receptor for IFN- $\gamma$ (unpublished observations). Type I IFNs, which are central to antiviral defenses, encompass a large family of antiviral cytokines that include multiple IFN- $\alpha$ proteins and a single IFN- $\beta$ protein $(10,11)$. Type I IFNs signal through a common receptor, IFN- $\alpha / \beta$ receptor (IFNAR), resulting in the expression of proinflammatory genes that inhibit viral replication and affect various aspects of immunity $(12,13)$. S. pneumoniae nasopharyngeal colonization has been shown to induce type I IFNs and IFN-inducible genes in mice (14). While the importance of type I IFNs to antiviral defense is well established, our understanding of their role in bacterial defense, especially for extracellular bacteria such as $S$. pneumoniae, remains incomplete.

Our laboratory has examined a number of bacterial and host factors that contribute to the establishment and clearance of pneumococcal colonization in mice. Once colonization of the URT is established, there is a gradual clearance of organisms along the mucosal surfaces of the nasal spaces. During primary 
A

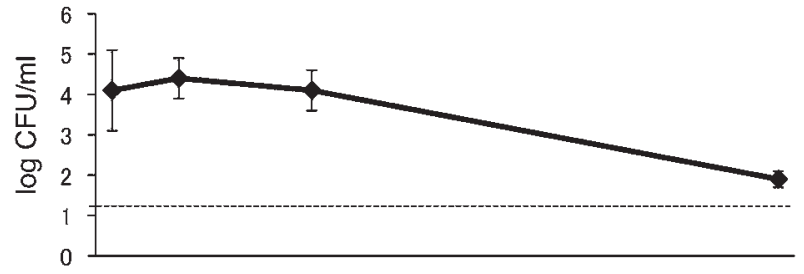

B

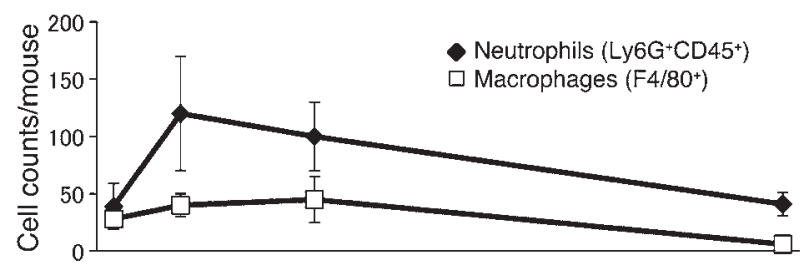

C

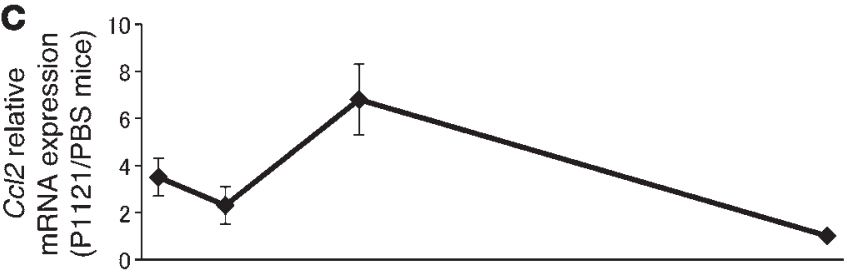

D

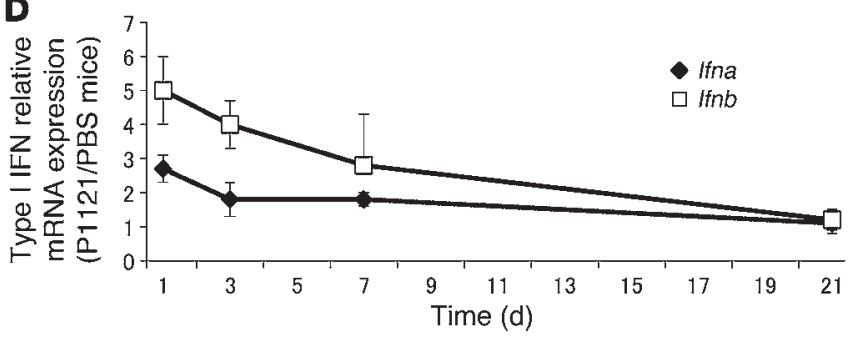

colonization, antibody and neutrophils have a minimal impact on clearance because the neutrophil influx resolves before specific antibody is generated $(15,16)$. The decline in the bacterial load requires the influx of monocytes/macrophages into the airway lumen (15). Recruitment of these cells correlates with the expression of the chemokine CCL2 and is deficient in mice lacking its receptor, CCR2, which is also required for early clearance $(15,17)$. Recognition of pneumococci by pattern recognition receptors TLR2 and Nod2 - which sense lipid-modified constituents and muramyl dipeptide-containing (MDP-containing) peptidoglycan fragments, respectively - contributes to the production of CCL2, the influx of macrophages, and bacterial clearance.

Here we show that influenza and pneumococcal coinfection of the URT results in synergistic stimulation of type I IFNs, which attenuates the production of CCL2 and impairs macrophage recruitment. These effects inhibit clearance and result in a higher bacterial load. We propose that altered colonization dynamics during influenza virus infection may play a role in the complications associated with coinfection that occur outside the nasopharynx.

\section{Results}

Pneumococcal nasopharyngeal colonization induces type IIFNs. The type I IFN response during murine pneumococcal colonization was investigated using strain P1121, a type 23F clinical isolate that does not cause invasive infection in mice (18). URT lavages were analyzed to evaluate the kinetics of colonization by quantitative

\section{Figure 1}

Type I IFNs are expressed during pneumococcal carriage. (A) URT lavages were analyzed for quantitative culture at the time points indicated, following intranasal inoculation of C57BL/6 WT mice with strain P1121. Dashed line represents the limit of detection. (B) URT lavages from colonized mice were analyzed by flow cytometry to quantify numbers of neutrophils and macrophages. (C) Levels of Cc/2 mRNA in URT tract lavages were determined using qRT-PCR at each time point and expressed relative to uncolonized mice. (D) Levels of type I IFN mRNA in URT lavages were determined using qRT-PCR at each time point and expressed relative to uncolonized mice. $n=10-15$ mice per time point. Values represent mean \pm SD.

culture and the cellular inflammatory response by flow cytometry. As previously documented, the density of P1121 decreased gradually over the observation period (Figure 1A), and the numbers of luminal neutrophils ( $\mathrm{Ly}_{6} \mathrm{G}^{+} \mathrm{CD} 45^{+}$population) and macrophages ( $\mathrm{F} 4 / 80^{+}$population) were maximal at day 3 and day 7 after inoculation, respectively (Figure 1B and ref. 15). The recruitment of macrophages correlated with the expression of the chemokine $\mathrm{Ccl} 2$ (Figure 1C). There was a moderate increase in mRNA for Ifna and Ifnb in the URT, with a maximal response at day 1 after inoculation and decline that paralleled the loss of colonization (Figure 1D).

Nasal poly-ICLC treatment impacts pneumococcal colonization. To ascertain the effect of additional stimulation of type I IFNs, we treated mice intranasally with a daily dose of the synthetic TLR3 ligand poly-ICLC or vehicle control following the establishment of pneumococcal colonization. As expected, poly-ICLC treatment induced higher levels of type I IFN mRNA in URT lavages of colonized mice (Figure 2A). Poly-ICLC treatment was sufficient to increase the mean pneumococcal colonization density by greater than 20 -fold at day 7 after inoculation (Figure 2B). An effect of poly-ICLC was not observed in Ifnar ${ }^{-}$mice, with a targeted deletion of the common type I IFN receptor, demonstrating that additional stimulation of type I IFNs was required for the poly-ICLC-mediated increase in colonization density. Poly-ICLC treatment was associated with an IFNdependent decrease in macrophage recruitment to the URT lumen at day 7 , the time point when the numbers of luminal macrophages per animal peaks (Figure 2C). The decreased macrophage recruitment correlated with lower expression of $C c l 2$ in poly-ICLC-treated mice (Figure 2E). There was no effect of poly-ICLC treatment on the numbers of luminal neutrophils or levels of mRNA for the chemokine KC, which triggers neutrophil recruitment (Figure 2, D and E). An effect of poly-ICLC on colonization was not seen with dosing every third day rather than daily. Because less frequent dosing stimulated much less type I IFN transcription (2.5- vs. 60-fold increase in IFN- $\beta$ mRNA transcription compared with PBS control at day 7), the effect of dose suggested that sustained high levels of type I IFN were required to impact clearance of colonization.

Synergistic production of the type I IFNs by influenza virus increases pneumococcal colonization density. Next, we examined whether coinfection with influenza virus has a similar effect on type I IFN expression and pneumococcal colonization. C57BL/6 mice were infected intranasally with the $P R 8$ strain of influenza A virus (median tissue culture infective dose $\left[\mathrm{TCID}_{50}\right]$ of 1,000$)$ a day after colonization was established with S. pneumoniae P1121 (Figure 3A). Virus-infected mice showed mild weight loss ( $<10 \%$ body weight) with a nadir at day 6 following viral inoculation (Figure 3B). At day 7 after challenge with influenza virus, PR8 was detected in the URT lavages at high titers (Figure 3C). Because virus and bacteria were administered intrana- 


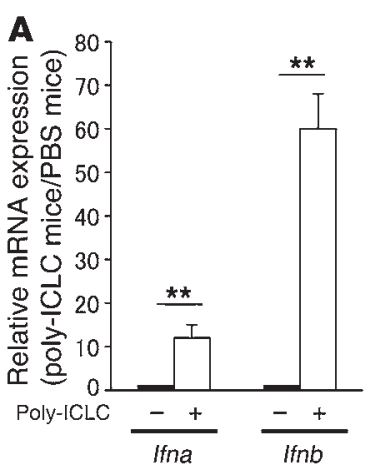

C

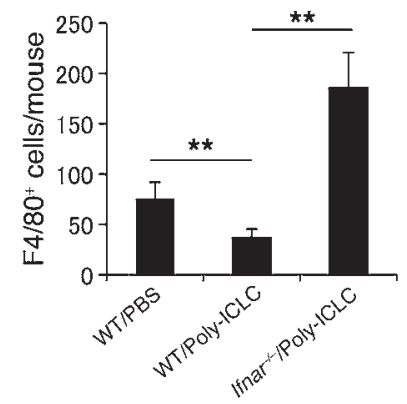

B

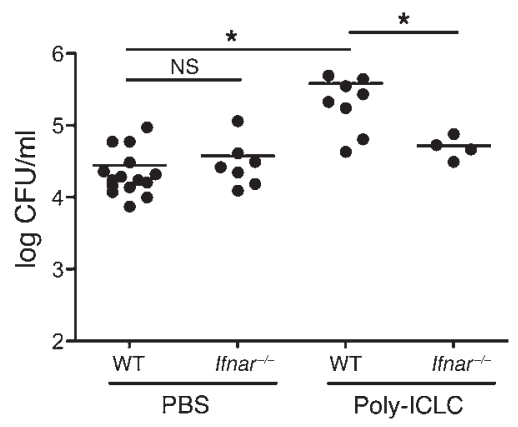

D

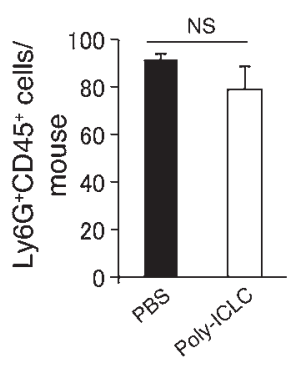

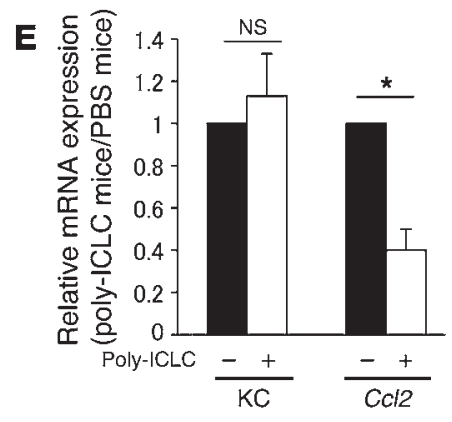

\section{Figure 2}

Stimulation of type I IFNs increased pneumococcal nasopharyngeal colonization. URT lavages from colonized WT and Ifnar-1- mice were treated with poly-ICLC (6 $\mu$ g i.n. daily for 7 days) and compared with PBS vehicle controls for (A) type I IFN mRNA expression in WT mice, (B) density of strain P1121 colonization, (C and D) cell counts in URT lavages analyzed by flow cytometry (F4/80+ macrophages and $\mathrm{Ly} 6 \mathrm{G}+\mathrm{CD} 45^{+}$neutrophils), and (E) chemokine $\mathrm{KC}$ and $\mathrm{Cc} / 2$ mRNA expression in WT mice. $n=9$ mice per group. Values represent mean \pm SD. Values are expressed relative to the PBS control group. ${ }^{*} P<0.05,{ }^{* *} P<0.01$. sally without anesthesia to avoid aspiration, only low levels of influenza and no pneumococci were detected in lung homogenates. Furthermore, there were no pathological differences in lungs sections with or without influenza virus challenge (Figure 3D), suggesting that influenza infection was largely limited to the URT.

As shown in Figure 3E, intranasal inoculation of $P R 8$ resulted in increased pneumococcal load at day 7 of bacterial infection. Coinfected mice showed a synergistic increase in IFN- $\beta$ mRNA expression compared with mice infected with either $S$. pneumoniae or influenza virus alone (Figure $3 \mathrm{~F}$ ). The synergistic increase in IFN- $\beta$ expression during coinfection was associated with an inhibition of macrophage, but not neutrophil, recruitment (Figure 3G), which correlated with decreased expression of $\mathrm{Ccl} 2$, but not $\mathrm{KC}$ (Figure $3 \mathrm{H}$ ). In contrast, in Ifnar/- mice there was no increase in the density of colonizing P1121 during coinfection with PR8 (Figure 4A). Likewise, there was no loss of macrophage recruitment or decrease in $\mathrm{Ccl} 2$ expression in coinfected Ifnar $/-$ mice (Figure 4, B and C). These results indicated that (a) concurrent influenza infection of the URT amplifies type I IFN production and (b) the increased pneumococcal colonization density requires type I IFN signaling. Additionally, we examined the relationship between increasing pneumococcal colonization density and development of disease. We used an intranasal inoculum of an invasive serotype $6 \mathrm{~A}$ pneumococcal strain with or without PR8 coinfection and compared survival. The rate of late-onset sepsis was higher in coinfected mice compared with mice receiving pneumococcal challenge alone (Figure 3I). These data indicated that the higher density of bacterial colonization induced by influenza virus may predispose to invasive disease and mortality.

In a separate set of experiments, we tested the effect of previously established viral infection on pneumococcal infection, a scenario commonly examined in models of secondary pneumonia. When strain P1121 was given on day 6 following intranasal administration of PR8 $\left(1,000 \mathrm{TCID}_{50}\right)$, there was no synergistic stimulation of type I IFNs or significant effect of antecedent viral infection on the density of pneumococcal colonization at day 7 (data not shown). Thus, the effect of influenza virus on pneumococcal colonization may be most relevant to concurrent infection.

The type I IFN response to $S$. pneumoniae requires macrophages and is Nod2 dependent. Because the bacterial contribution to type I IFN expression was necessary to affect colonization and disease, the mechanism for the type I IFN stimulation by $S$. pneumoniae was investigated further. The CCL2-CCR2-dependent recruitment of monocytes/macrophages contributed to the sustained production of type I IFNs. At day 7 after inoculation, $\mathrm{C} c 2^{-1-}$ mice showed lower levels of IFN- $\beta$ transcription (Figure $5 \mathrm{~A}$ ). As shown in Figure 5B, there was no difference between $T l r 2^{+/+}$and $T l r 2^{-/-}$mice in the type I IFN mRNA expression in URT lavages at 3 days after infection with strain P1121. In contrast, the expression of IFN- $\beta$ mRNA was no longer stimulated in Nod2 ${ }^{-/-}$colonized mice (Figure 5C). This requirement for Nod 2 for the stimulation of IFN- $\beta$ was also observed in peritoneal macrophages treated ex vivo with P1121. Accordingly, $\mathrm{Nod}_{2}{ }^{-/-}$mice no longer showed a synergistic increase in IFN- $\beta$ or suppression of $C c l 2$ mRNA levels in URT lavages during coinfection (Figure 5, D and E). In the absence of the synergistic increase in IFN- $\beta$ there was no increased pneumococcal colonization in the presence of influenza virus in $\mathrm{Nod}^{-/-}$mice (Figure $5 \mathrm{~F}$ ). We also examined the role of specific transcription factors in the type I IFN response to $S$. pneumoniae. IFN- $\beta$ transcription in P1121-stimulated bone marrow-derived macrophages was reduced in $\mathrm{Irf3}^{-/-}$and $\mathrm{Irf5} 5^{-/-}$macrophages (Figure 5G).

Type I IFN inhibits production of CCL2 in peritoneal macrophages. Both poly-ICLC treatment of colonized mice and coinfection inhibited the expression of CCL2 and influx of luminal macrophages, suggesting these effects required a synergistic type I IFN response. To further explore this question, we pretreated peritoneal macrophages isolated from WT mice with poly-ICLC or recombinant IFN- $\beta$ and 
A

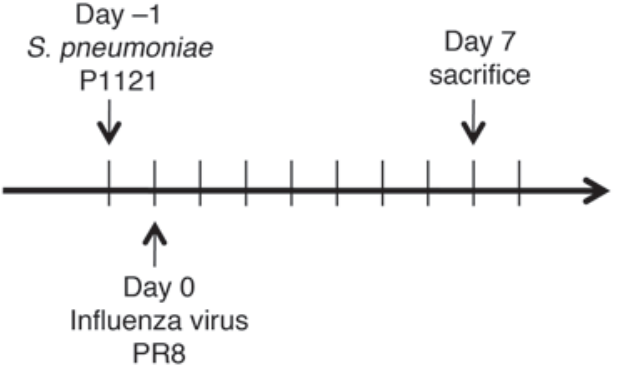

D P1121 alone

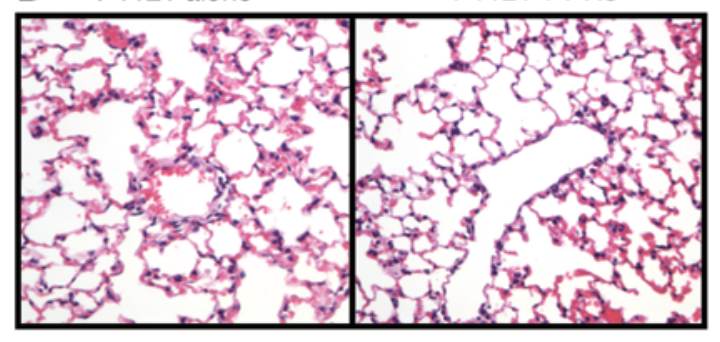

B

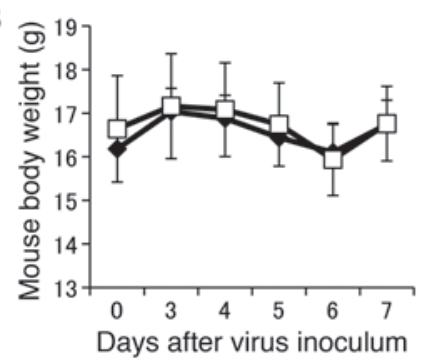

E

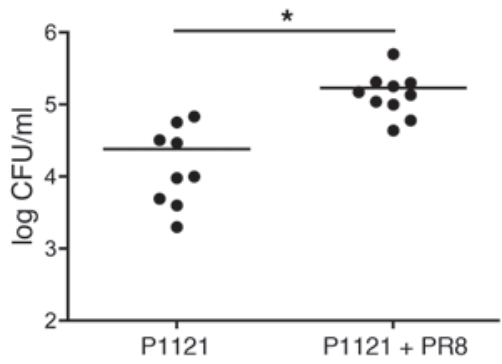

C

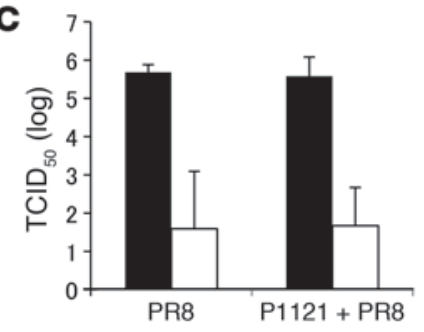

$\mathbf{F}$

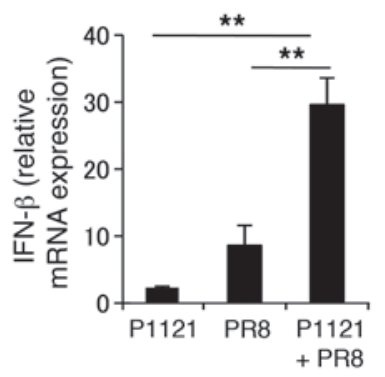

G

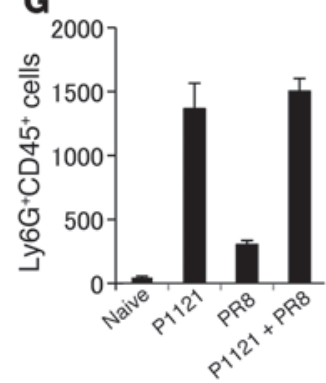

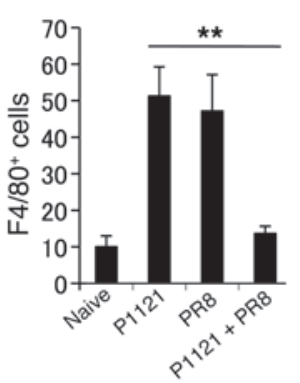

H
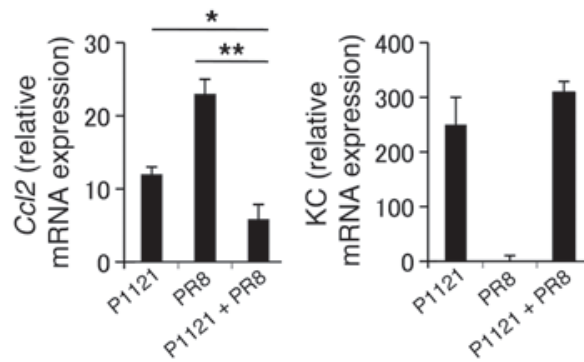

I

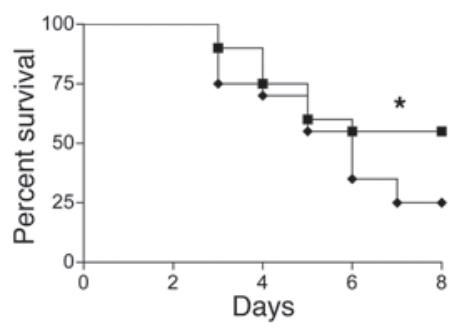

\section{Figure 3}

Coinfection with influenza virus induces synergistic IFN- $\beta$ expression and enhances pneumococcal nasopharyngeal colonization. (A) Experimental protocol for coinfection model. WT mice were given an intranasal inoculation with S. pneumoniae (P1121 strain, $10^{7}$ CFU) or PBS, followed a day later by an intranasal inoculation with influenza virus (PR8 strain, 1,000 TCID 50 ) or PBS. (B) Mice infected with PR8 strain were monitored for body weight (white squares, P1121+PR8; black diamonds, PR8 alone). (C) Titer of influenza virus in singly infected and coinfected mice was calculated based on viral RNA detected in URT lavages (black bars) and lung homogenates (white bars). (D) H\&E staining of representative lung tissue sections from mice infected with $S$. pneumoniae alone and coinfected with influenza virus. Original magnification, $\times 200$. Horizontal lines indicate mean values. (E) The density of pneumococcal colonization was measured in singly infected and coinfected mice at day 7 . (F) Levels of IFN- $\beta$ mRNA in URT lavages from singly infected and coinfected mice were examined by qRT-PCR. (G) Numbers of F4/80+CD45+ macrophages and $\mathrm{Ly} 6 \mathrm{G}+\mathrm{CD} 45^{+}$neutrophils recruited to the URT were analyzed by flow cytometry from uninfected, singly infected, and coinfected mice. (H) Levels of $\mathrm{Cc} / 2$ and KC mRNA in URT lavages from singly infected and coinfected mice as determined by qRT-PCR. (I) Survival rates of mice infected with serotype 6A pneumococcus alone (squares; $n=20$ ) and serotype 6A pneumococcus coinfected with PR8 (diamonds; $n=20$ ). Values represent mean $\pm \mathrm{SD} .{ }^{*} P<0.05,{ }^{*} P<0.01$.

then stimulated them with strain P1121. The stimulation of CCL2 protein and $C c l 2$ mRNA expression by P1121 was inhibited by both poly-ICLC and IFN- $\beta$ (Figure 6, A and B). In contrast, the inhibitory effect of poly-ICLC and IFN- $\beta$ was not seen in macrophages from Ifnar ${ }^{-1}$ mice. We had previously shown that the CCL2 response to S. pneumoniae was NF-кB-dependent and required sensing of peptidoglycan by Nod2 (17). To examine directly the inhibitory effect of poly-ICLC and IFN- $\beta$ on Nod 2 signaling, we used HEK293T cells transfected with a Nod2-expressing plasmid or empty vector control and NF-KB reporter plasmid. HEK293T cells were preincubated with poly-ICLC or IFN- $\beta$ and then stimulated with the synthetic Nod2 ligand MDP (Figure 6C). NF- $\mathrm{KB}$ activation was inhibited by
poly-ICLC or IFN- $\beta$, providing a direct demonstration that type I IFNs are sufficient to inhibit Nod2 signaling.

Type I IFN induction by S. pneumoniae is dependent on a pore-forming toxin, pneumolysin. An additional factor in Nod2-mediated responses to $S$. pneumoniae is the expression of the pneumococcal pore-forming toxin pneumolysin (17). We compared levels of IFN- $\beta$ mRNA in peritoneal macrophages stimulated with WT, ply- (pneumolysin gene deleted), and ply W433F $_{\text {(pneumolysin point }}$ mutant defective in pore formation) $S$. pneumoniae. As shown in Figure $7 \mathrm{~A}$, IFN- $\beta$ mRNA expression was reduced in macrophages stimulated with either mutant strain and restored when ply was replaced in the deletion mutant. In vivo, neither mutant showed 

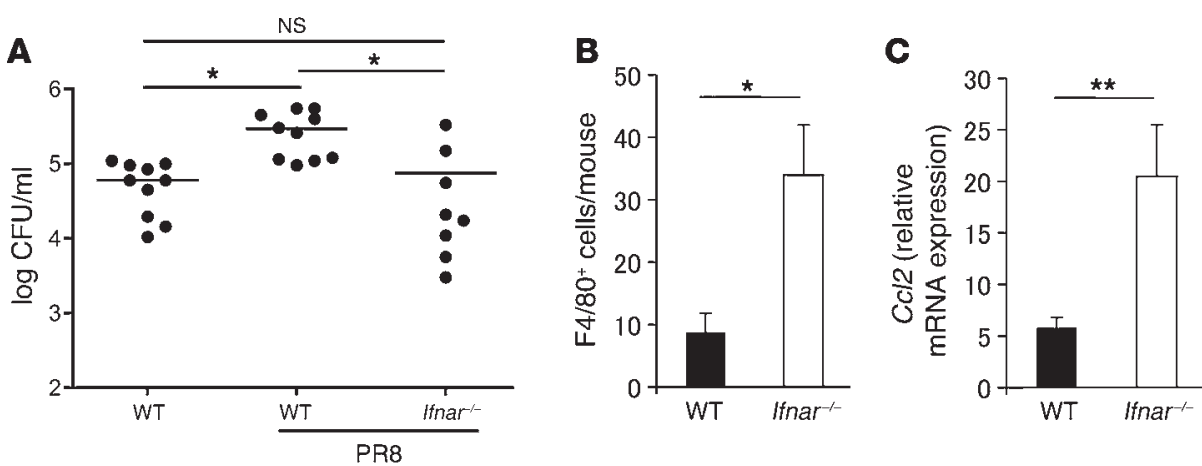

Figure 4

The effect of coinfection of influenza strain PR8 on colonization by pneumococcal strain P1121 is dependent on type I IFNs. (A) Density of pneumococcal colonization in URT lavages from singly infected and coinfected WT and Ifnar ${ }^{-1-}$ mice at day 7 after challenge with influenza virus. Horizontal lines indicate mean values. (B) Numbers of $\mathrm{F} 4 / 80^{+}$macrophages recruited to URT in coinfected WT and Ifnar-1- mice. (C) Levels of Ccl2 mRNA expression in URT lavages in coinfected WT and Ifnar ${ }^{-1}$ mice were determined by qRT-PCR. Values represent mean $\pm \mathrm{SD} .{ }^{\star} P<0.05,{ }^{\star \star} P<0.01$.

synergistic induction of IFN- $\beta$ or increased colonization density during coinfection with influenza virus (Figure 7, B and C). This demonstrated that pore formation by pneumolysin plays a crucial role in the type I IFN-dependent exacerbation of pneumococcal colonization by influenza virus.

\section{Discussion}

Bacterial infection of the respiratory tract is common, often occurs in the setting of antecedent or concurrent viral infection, and typically involves the opportunistic pathogens that colonize the upper airway. In this study, we addressed whether influenza A virus impacts colonization, the initial step in the interaction of S. pneumoniae with its host, using a mouse model of URT coinfection. Our results demonstrated that concurrent influenza infection increased the density of colonizing pneumococci. This finding correlated with clinical surveys using quantitative nasal culture to assess the effect of concurrent viral infection in children and experimental influenza infection studies in adults - both of which showed increased isolation of $S$. pneumoniae from nasal cultures (2, 19). An increased burden of colonizing pneumococci could be an independent risk factor for the development of bacterial disease. Since the IFN response is common to many viruses, our report offers a mechanistic explanation for the association of acute viral infections and the increased rates of pneumococcal disease and its markedly seasonal pattern $(5,20)$. The consequence of increased bacterial load was also demonstrated in our study when an invasive strain was analyzed and, in the setting of coinfection, led to a higher rate of sepsis. An increased density of colonizing organisms during coinfection could also contribute to increased host-to-host transmission, as suggested in surveillance studies and recently demonstrated in animal models $(19,21,22)$.

Previous studies examining coinfection in mice have focused on the LRT and the secondary bacterial pneumonia that occurs many days after viral infection is initiated. Our coinfection model, in contrast, avoided direct inoculation into the LRT and was associated with minimal viral and bacterial titers and pathology in the lung. Viral infection limited to the URT impacted concurrent or recently established bacterial colonization, and there was no detectable effect on colonization if the bacterial challenge was given during the recov- pneumococcus could not be explained by changes in the normal mouse flora (data not shown). Joyce et al. had previously described a microarray analysis of nasal-associated lymphoid tissue (NALT) and identified several hundred genes whose expression was significantly changed during pneumococcal carriage, including the induction of type I IFN and type I IFN-induced genes (14). In our study, this response did not seem to be sufficient to impact colonization, since pneumococcal colonization alone was unaltered in Ifnar $/-$ mice. Further stimulation of type I IFNs in S. pneumoniae-colonized mice using treatment with intranasal poly-ICLC, however, resulted in increased colonization density that was not observed in Ifnar ${ }^{-1}$ mice and therefore was dependent on type I IFN signaling. Moreover, this synergistic increase in type I IFNs was seen during coinfection with influenza virus and was necessary and sufficient to promote bacterial colonization. Thus, we focused our analysis on how this synergistic type I IFN response was induced and why it enhanced colonization.

We recently showed that production of chemokine CCL2 and its recognition by CCR 2 drive the influx of macrophages into the lumen of the URT during pneumococcal colonization $(15,17)$. In the lung, overexpression of CCL2 is sufficient to improve pneumococcal clearance (24). The expression of CCL2 and influx of F4/80 macrophages into the URT was inhibited during coinfection or by treatment with poly-ICLC. Accordingly, Zimmerer et al. profiled STAT1-dependent genes regulated by IFN- $\alpha$ and showed a suppression of Ccl2 expression (25). We observed no effect on URT expression of the chemokine $\mathrm{KC}$ or on neutrophil recruitment, which contrasts with the report by Shahangian et al. showing that type I IFNs inhibited KC expression and lead to attenuated neutrophil responses during secondary pneumococcal pneumonia (26). Our results also contrast with those of Antonelli et al., who showed that the increased pulmonary burden of Mycobacterium tuberculosis following intranasal poly-ICLC treatment was associated with a CCR2-dependent increase in a $\mathrm{F} 4 / 80^{+}$cell population permissive for infection (27). Additionally, Jia et al. reported that the induction of CCL2 and monocyte recruitment in response to Listeria monocytogenes infection was promoted by type I IFN signaling (28). Differences in the anatomic site and type of infecting agent may account for the varied effects of type I IFNs among these studies. The production of CCL2 during S. pneumoniae colonization is also 

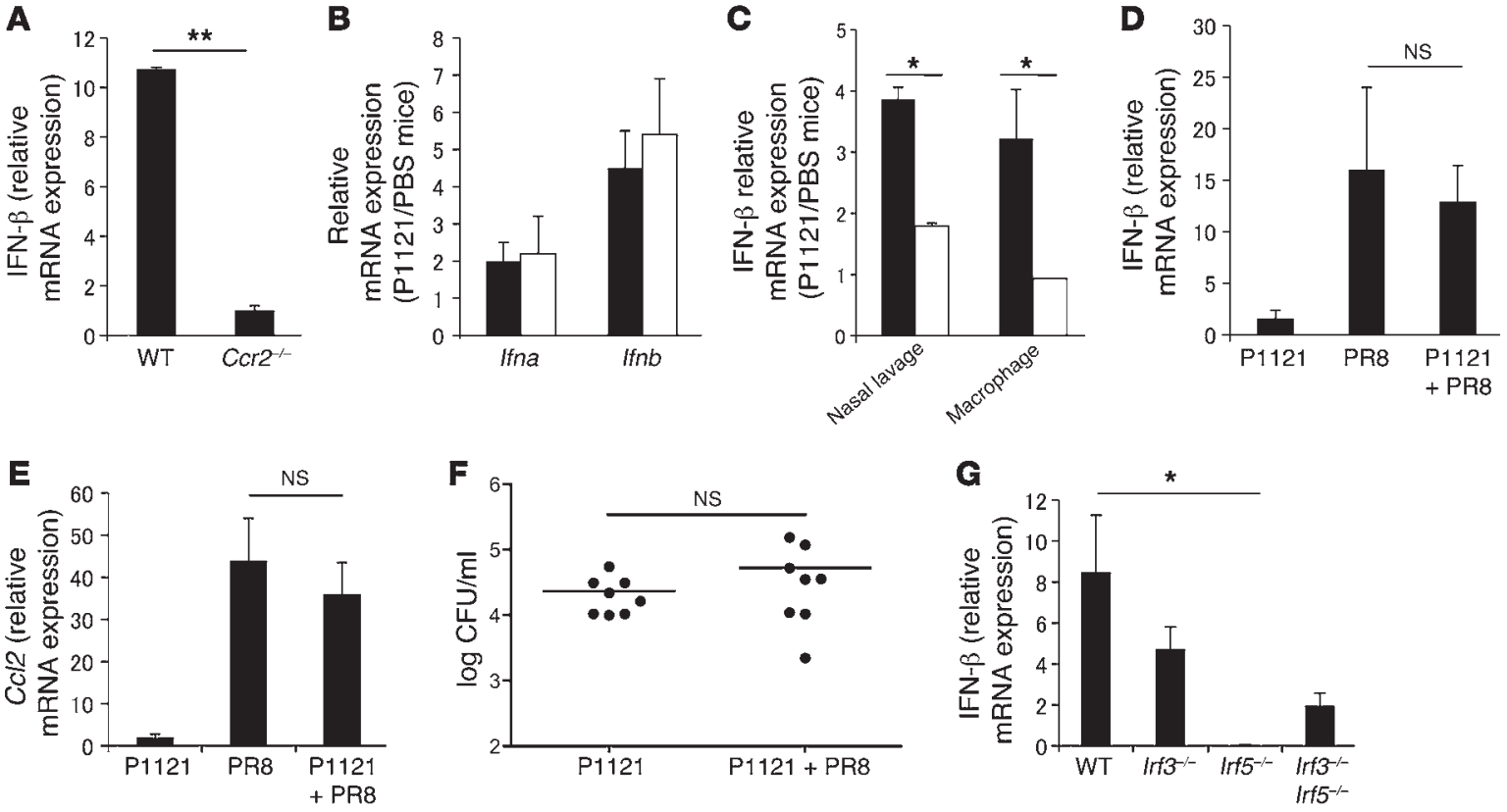

Figure 5

Macrophages and Nod2 contribute to production of type I IFN by S. pneumoniae. (A) The levels of type I IFN mRNA in URT lavages of WT and $\mathrm{Ccr}^{-/-}$mice were analyzed 7 days after intranasal infection with strain P1121 by qRT-PCR. (B) The levels of type I IFN mRNA in URT lavages of WT (black bars) and TIr2-/- (white bars) mice were analyzed 3 days after intranasal infection with strain P1121. (C) The levels of IFN- $\beta$ mRNA in URT lavages of WT (black bars) and Nod2-/- (white bars) mice were analyzed 3 days after intranasal infection with strain P1121. Levels of IFN- $\beta$ mRNA in peritoneal macrophages from WT and Nod2-/- mice were examined 12 hours after stimulation with heat-killed strain P1121. Levels of (D) IFN- $\beta$ and (E) Ccl2 mRNA in URT lavages of singly infected and coinfected Nod2--- mice determined by qRT-PCR. (F) Density of pneumococcal colonization in URT lavages of singly infected and coinfected $\mathrm{Nod}^{-/-}$mice day 7 after challenge with influenza virus PR8. Horizontal lines indicate mean values. (G) The levels of type I IFN mRNA in bone marrow-derived macrophages from WT, Irf3-/- Irf5 $5^{-/-}$, Irf3 ${ }^{-1-I r f 5^{-/-}}$mice were examined 12 hours after stimulation with heat-killed strain $\mathrm{P} 1121$. Values represent mean $\pm \mathrm{SD}$. ${ }^{\star} P<0.05,{ }^{\star \star} P<0.01$.

affected by the Th17 response (15). IL-17A production is required for mucosal immunity to $S$. pneumoniae and is Nod2 dependent $(15,17)$. However, Il17a transcription in response to P1121 was similar with and without PR8 (data not shown).

Our in vivo observations that synergistic stimulation of type I IFNs inhibits expression of CCL2 correlated with the effects of IFN- $\beta$ and poly-ICLC on the transcription and production of CCL2 by ex vivo macrophages. Thus, macrophages could be both the source of CCL2 and the target of type I IFNs, which act to block the CCR2-dependent recruitment of additional macrophages to the site of infection. By day 7 after infection, pneumococci in the URT are seen in association with macrophages, and the loss of URT macrophages inhibits the clearance of pneumococcal colonization (15). Thus, the reduced numbers of URT macrophages in the setting of synergistic stimulation of type I IFNs could explain the increased colonization density seen on day 7 after infection.

There is a growing appreciation for the ability of bacteria to stimulate a type I IFN response, although most of the studies have emphasized intracellular pathogens. A number of pathways have been proposed (29). The pneumococcus is an extracellular pathogen, but it expresses a pore-forming toxin, pneumolysin, that can allow access of microbial products for sensing by cytosolic pathways $(30,31)$. In addition, TLR4-dependent responses to pneumolysin have been described, and although TLR4 signaling through its adaptor TRIF stimulates type I IFN expression via the Irf3 pathway, the effect of pneumolysin requires the adaptor Myd88 (32). The stimulation of type I IFNs by bacteria, including S. pneumoniae, has mainly been attributed to recognition of nucleic acids by cytosolic sensors, which act through the downstream signaling adaptors to induce type I IFN in a Irf-dependent manner $(33,34)$. In addition, recognition of bacterial cell wall fragments by the cytosolic sensor Nod2, which leads to Rip2-dependent NF-кB activation, has been shown to induce type I IFNs $(35,36)$. In the case of $M$. tuberculosis, induction of type I IFNs requires sensing of its peptidoglycan by Nod 2 and is dependent on both Irf3 and Irf5 (35). In our colonization model, the dominant pathway for pneumococcal induction of IFN- $\beta$ required sensing by the Nod2-dependent pathway. Moreover, in bone marrow-derived macrophages, Irf5 was needed for the IFN- $\beta$ response to $S$. pneumoniae. A further observation was that the NF- $\kappa \mathrm{B}$ activation in response to MDP, the peptidoglycan fragment sensed by Nod2, was completely blocked by either poly-ICLC or IFN- $\beta$, confirming the effects of type I IFN signaling on Nod2-dependent gene expression. However, the components of the signaling pathway downstream of Nod 2 affected by the type I IFN response to $S$. pneumoniae have not yet been defined. It will be important to understand how the Nod2-dependent pathway synergizes with the influenza-stimulated pathway, since enhanced bacterial infection required their combined effects.

\section{Methods}

Bacterial strains and culture. S. pneumoniae strains were grown in tryptic soy medium as described previously (37). Strain P1121, a type 23F isolate obtained from a study of experimental human colonization, was selected for 

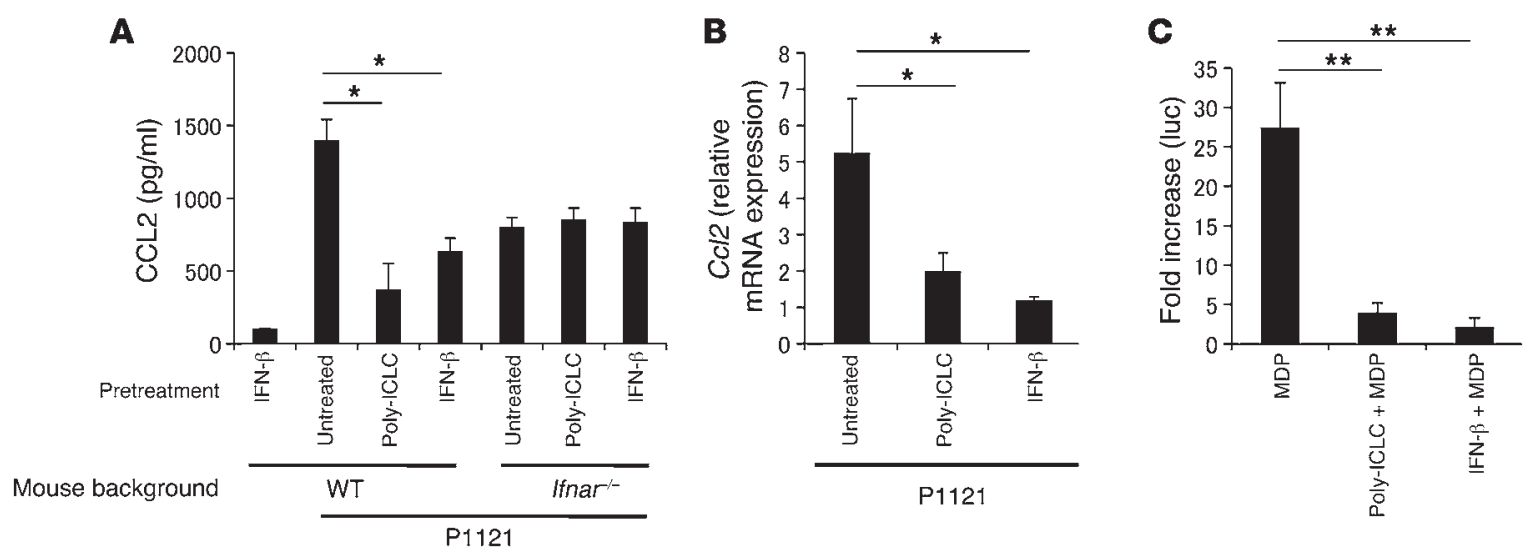

Figure 6

IFN- $\beta$ inhibits production of CCL2 in peritoneal macrophage stimulated with heat-killed S. pneumoniae. (A) Effect of treatment of peritoneal macrophages with poly-ICLC or IFN- $\beta$ prior to stimulation with heat-killed strain P1121 on CCL2 production in culture supernatants as assessed by ELISA. (B) Levels of Cc/2 mRNA expression in P1121-stimulated peritoneal macrophages with the pretreatment indicated as analyzed by qRT-PCR. (C) Luciferase assay comparing NF-кB activity in Nod2-expressing 293T cells treated with MDP with or without pretreatment with poly-ICLC or IFN- $\beta$. Values are relative to empty vector controls and expressed as mean $\pm \mathrm{SD}$. ${ }^{*} P<0.05,{ }^{*} P<0.01$.

its ability to efficiently colonize the murine nasopharynx $(15,16,18)$. The construction of defined pneumolysin mutants of P1121 using bicistronic positively and negatively selectable Janus cassette technology was previously described and included: (a) ply-, a strain containing an unmarked, complete in-frame deletion of the pneumolysin gene; (b) $p y_{W 433 F}$, a strain with the deletion restored with a point mutation previously shown to reduce the efficiency of pore formation $95 \%-99 \%$, and (c) $\mathrm{ply}^{+}$, a strain with the deletion restored with the entire ply gene $(30,38)$. All strains were passaged intranasally in mice before preparation of frozen stocks $\left(-80^{\circ} \mathrm{C}\right)$.

Mice. Wild-type C57BL/6 and congenic Nod2 $2^{-/}, \mathrm{Tlr}^{-/-}$, and $\mathrm{Ccr} 2^{---}$mice were from The Jackson Laboratory. Ifnar ${ }^{--}$mice lacking the IFN- $\alpha \beta$ receptor were provided by S. Weiss (University of Pennsylvania) (39). Animal studies were conducted according to protocols approved by the IACUC of the University of Pennsylvania. Mice included both males and females inoculated at age 5-8 weeks. At the designated time point, mice were sacrificed by $\mathrm{CO}_{2}$ asphyxiation.

Pneumococcal colonization. S. pneumoniae strain P1121 was grown until mid-log phase was reached $\left(\mathrm{OD}_{620}=0.5\right)$, pelleted, and resuspended in PBS to a density of $10^{9} \mathrm{CFU} / \mathrm{ml}$. For colonization studies, $10 \mu \mathrm{l}\left(\sim 10^{7} \mathrm{CFU}\right)$ of the pneumococcal suspension was instilled into the nares of each mouse without anesthesia. At the time point specified, mice were sacrificed by $\mathrm{CO}_{2}$ asphyxiation, and the trachea was exposed and cannulated for instillation of $200 \mu \mathrm{l}$ sterile PBS. The lavage fluid exiting the nares was collected, and S. pneumoniae was quantified by plating of serial dilutions of the nasal lavages onto tryptic soy agar plates supplemented with catalase (4,741 U/plate) (Worthington Biochemical Corp.) and incubated overnight at $37^{\circ} \mathrm{C}$ in a $5 \%$ $\mathrm{CO}_{2}$ atmosphere. Neomycin $(20 \mu \mathrm{g} / \mathrm{ml})$ was added to plates to inhibit the growth of contaminants. Where specified, for quantitative RT-PCR (qRTPCR) analysis, a second nasal lavage was collected using $600 \mu \mathrm{l}$ RLT buffer (QIAGEN) with $1 \% \beta$-mercaptoethanol and stored at $-80^{\circ} \mathrm{C}$ until use.

Where indicated, WT mice were challenged intranasally with $1 \times 10^{6}$ to $1 \times 10^{7} \mathrm{CFU}$ of strain P1547 (S. pneumoniae serotype 6A, mouse virulent, clinical isolate from blood) (40) with or without sequential influenza virus infection. Mice were observed for signs of sepsis over an 8-day period after challenge, and following euthanasia spleens were cultured to confirm the presence of pneumococcal bacteremia.

Microbiota depletion. To determine the effect of the microbiota on the induction of type I IFN, we depleted normal flora from mice by addition
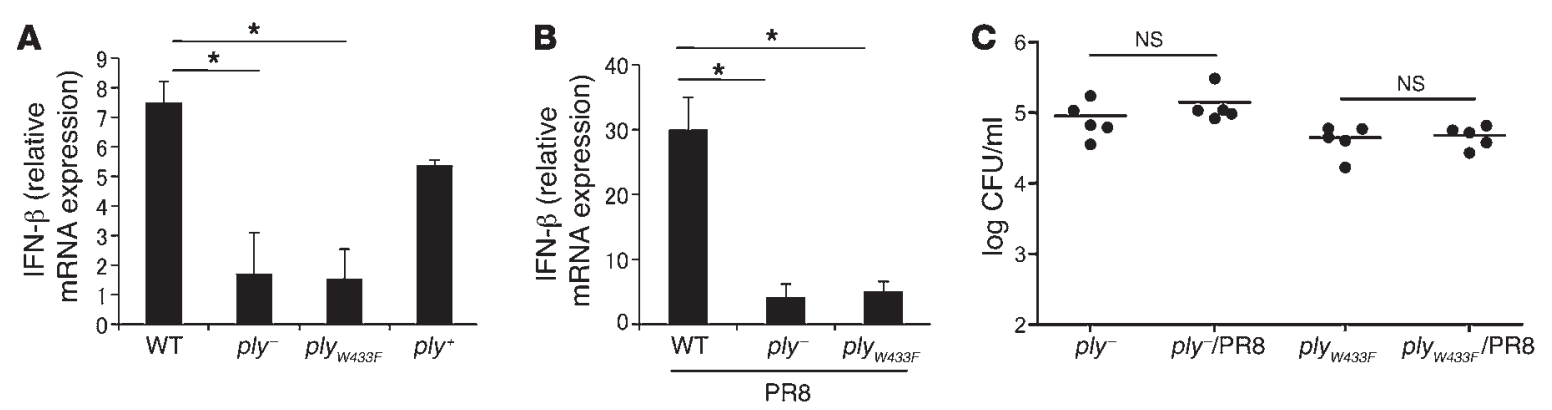

\section{Figure 7}

Pore formation by Ply contributes to type I IFN expression. (A) Levels of IFN- $\beta$ mRNA determined by qRT-PCR in peritoneal macrophages following stimulation with heat-killed P1121 and isogenic mutants with a deletion of the entire ply (ply-), with a W433F point mutant defective in pore formation $\left(p / y_{w 433}\right)$, or with $p l y$ restored in the deletion mutant $\left(p / y^{+}\right)$. (B) The levels of IFN- $\beta$ mRNA in URT lavages obtained from the mice coinfected with PR8 and the pneumococcal strain indicated were determined by qRT-PCR. (C) Comparison of the colonization density in URT lavages of WT mice singly infected or coinfected with the pneumolysin mutant indicated and PR8 at day 7 after influenza virus infection. Values represent mean \pm SD. Horizontal lines indicate mean values. ${ }^{*} P<0.05$. 
of antibiotics (ampicillin $1 \mathrm{~g} / \mathrm{ml}$, Mediatech; neomycin sulfate $1 \mathrm{~g} / \mathrm{ml}$, Calbiochem; metronidazole $1 \mathrm{~g} / \mathrm{ml}$, Fluka; and vancomycin $0.5 \mathrm{~g} / \mathrm{ml}$, SigmaAldrich) in drinking water for 7 days (41).

Whole-lung preparations. Whole lungs were removed and homogenized in $500 \mu \mathrm{l}$ PBS for quantitative bacterial culture. For RNA extraction from lung tissue, a portion of the lung was placed directly into $600 \mu \mathrm{l}$ of RLT buffer with $1 \% \beta$-mercaptoethanol and kept on ice. Lungs were homogenized using a QIAshredder Column (QIAGEN). For pathological analysis, whole lungs obtained from P1121-colonized mice with or without influenza virus were paraffin embedded and processed for H\&E staining.

Influenza virus infection and quantification. Mouse-adapted influenza virus strain A/Puerto Rico/8/34 (H1N1) (PR8; a gift from Peter Palese, Mount Sinai Hospital, New York, New York, USA) was grown in the allantoic fluid of embryonated eggs. For intranasal inoculation, a dose of 1,000 TCID $_{50}$ live virus in $20 \mu \mathrm{l}$ of Delbecco's PBS was given without anesthesia. Influenza infection was then monitored by daily measurement of body weight and viral titer calculated based on total viral RNA quantified by RT-PCR from both nasal lavages and lung homogenates (42).

Flow cytometry. Nasal lavages were pooled from at least 5 mice and the cells pelleted at $1,200 \mathrm{~g}$ for 5 minutes at $4{ }^{\circ} \mathrm{C}$. The pellets were washed and then resuspended in PBS with 1\% BSA. Fluorophore-conjugated antibodies recognizing different host cell surface proteins were incubated with cells on ice for 45 minutes. Typical final dilutions of the antibodies included 1:150 for PE-CD45 (BD) and APC-F4/80 (eBioscience) antibodies and 1:100 for FITC-Ly6G antibody (BD). All samples were resuspended in PBS with $1 \%$ BSA and analyzed on a BD FACSCalibur flow cytometer. All flow cytometry data were analyzed using FlowJo Mac, version 8.1.1 (TreeStar).

Poly-ICLC and in vivo treatment. Polyinosinic-polycytidylic acid condensed with poly-L-lysine (poly-ICLC, Hiltonol), a synthetic analog of viral dsRNA, was supplied by Oncovir Inc. Mice were inoculated intranasally with polyICLC ( $6 \mu \mathrm{g}$ in $20 \mu \mathrm{l} \mathrm{PBS} /$ mouse) with 3 doses (days -1, 2, and 5) or daily for a week, starting 1 day before $S$. pneumoniae challenge (27). Control animals received an equivalent volume of PBS.

Isolation and culture of peritoneal macrophages. Three days after intraperitoneal injection of $2 \mathrm{ml}$ of $4 \%$ sterile thioglycolate medium (Sigma-Aldrich), peritoneal macrophages were isolated by peritoneal lavage using Hank's buffer (without $\mathrm{Ca}^{2+}$ and $\mathrm{Mg}^{2+}$ ) with $0.1 \%$ gelatin. Contaminating erythrocytes, granulocytes, and dead cells were removed by density gradient centrifugation for 45 minutes at $800 \mathrm{~g}$ using MONO-POLY resolving medium according to the manufacturer's protocol (MP Biomedicals). Purified peritoneal macrophages were washed 3 times and cultured in Dulbecco's modified Eagle medium containing $10 \%$ FCS with $100 \mathrm{U} / \mathrm{ml}$ penicillin and $100 \mu \mathrm{g} / \mathrm{ml}$ streptomycin overnight before use in stimulation assays. Recombinant IFN- $\beta$ (final concentration $10^{4} \mathrm{U} / \mathrm{ml}$ ) or poly-ICLC (final concentration $25 \mu \mathrm{g} / \mathrm{ml}$ ) was added to the medium 30 minutes before stimulation with $10^{8} \mathrm{CFU}$ of strain P1121 that was heat killed at $65^{\circ} \mathrm{C}$ for 30 minutes. Supernatants for CCL2 ELISA were collected 24 hours later. Total cellular RNA was extracted from macrophages 12 hours after stimulation using the RNeasy Mini Kit (QIAGEN). No cytotoxicity was observed following stimulation with strain P1121 or poly-ICLC or IFN- $\beta$ treatment, as measured using the Cytotoxicity Detection Kit (LDH) (Roche) according to the manufacturer's protocol.

Isolation and culture of bone marrow-derived cells. Femurs and tibias isolated from mice of the indicated genetic backgrounds were provided by Kate Fitzgerald (University of Massachusetts Medical School, Worcester, Massachusetts, USA). Bones were washed with 70\% ethanol and then Hank's buffer (without calcium or magnesium). Bone marrow was isolated by flushing bones with macrophage differentiation media: DMEM, 20\% FCS, $30 \% \mathrm{~L}$ cell-conditioned medium, $100 \mathrm{U} / \mathrm{ml}$ penicillin, and $100 \mu \mathrm{g} / \mathrm{ml}$ streptomycin. Bone marrow cells were cultured 7 days in differentiation media at $37^{\circ} \mathrm{C}, 5 \% \mathrm{CO}_{2}$ in a humidified atmosphere. Cells were collected by chill- ing cultures on ice and washing with ice-cold PBS plus $2 \mathrm{mM}$ EDTA, then seeded at a density of $5 \times 10^{5}$ cells $/ \mathrm{ml}$ and incubated overnight prior to stimulation. Macrophage differentiation was confirmed by flow cytometry. Macrophages were stimulated 12 hours with $10^{8}$ heat-killed bacteria, and total cellular RNA was extracted using an RNeasy Mini Kit (QIAGEN). IFN- $\beta$ transcription was detected by qRT-PCR, and transcription of $\mathrm{NF}-\kappa \mathrm{B}-$ dependent cytokines was confirmed in all macrophage genetic backgrounds. No cytotoxicity was observed following stimulation or cytochalasin D treatment, as measured using the Cytotoxicity Detection Kit (LDH) according to the manufacturer's protocol (Roche).

Luciferase assay. HEK293T cells were cultured and transfected as previously described (41). Transfection reagents were washed off after 4 hours, and fresh media was added to cells. Transfected cells were pretreated with poly-ICLC (final concentration $25 \mu \mathrm{g} / \mathrm{ml}$ ) or IFN- $\beta$ (final concentration $10^{4} \mathrm{U} / \mathrm{ml}$ ) 30 minutes before MDP (98.5 ng) stimulation. Cells were stimulated for 20 hours, and luciferase expression was measured using the Luciferase Assay System (Promega) according to the manufacturer's protocol. Data were expressed as a fold difference in relative luciferase units relative to the empty vector control.

qRT-PCR. The epithelium lining the URT was harvested, and qRT-PCR was performed as previously described (43). One to $1.5 \mu \mathrm{g}$ RNA was used for reverse transcription using a High-Capacity cDNA Reverse Transcription Kit (Applied Biosystems) with $20 \mathrm{U}$ RNase inhibitor (Promega). One microliter of cDNA was used as template with $0.5 \mu \mathrm{M}$ primers and SYBR Green PCR Master Mix in a 20- $\mu 1$ reaction (Applied Biosystems). Standard runs were carried out using the StepOnePlus Real-Time PCR system (Applied Biosystems). Quantitative comparison was obtained through the $\triangle \Delta C_{T}$ method. Primers used were as follows: GAPDH-F: $5^{\prime}$-TGCACCACCAACTGCTTAG-3'; GAPDH-R: 5'-GGATGCAGGGATGATGTTC-3' (44); CCL2-F: 5'-AGCTCTCTCTTCCTCCACCAC-3'; CCL2-R: 5'-CGTTAACTGCATCTGGCTGA-3'; IFN- $\alpha$-F: 5'-TCTGATGCAGCAGGTGGG-3'; IFN- $\alpha$-R: $5^{\prime}$-AGGGCTCTCCAGACTTCTGCTCTG-3' (44); IFN- $\beta$-F: $5^{\prime}$ GCACTGGGTGGAATGAGACT-3'; IFN- $\beta$-R: $5^{\prime}$-AGTGGAGAGCAGTTGAGGACA-3' (44); mKC-F: 5'-CTGGGATTCACCTCAAGAACATC-3'; mKC-R: 5'-CAGGGTCAAGGCAAGCCTC-3'; IL-17A-F: 5'-TCTCATCCAGCAAGAGATCC-3'; IL-17A-R: 5'-AGTTTGGGACCCCTTTACAC-3' The detection of influenza virus RNA was performed by qRT-PCR with Taqman probe. Primers and probe used were as follows: PA-F: 5'-CGGTCCAAATTCCTGCTGAT-3'; PA-R: 5'-CATTGGGTTCCTTCCATCCA-3'; PA probe: 6FAM-CCAAGTCATGAAGGAGAGGGAATACCGCT-TAMRA.

ELISA. CCL2 ELISA kits were purchased from R\&D Systems. Cytokine levels were measured according to the manufacturer's protocol.

Statistics. All data were analyzed using StatView software (Abacus Concepts). The significance of differences between or among groups was examined using ANOVA followed by Dunn's post-tests. Statistical comparison of survival was made by the Fisher's exact test (GraphPad Prism 4). P values less than 0.05 were considered significant.

\section{Acknowledgments}

We thank E.J. Wherry and B. Laidlaw for measuring viral titers. This work was supported by grants from the U.S. Public Health Service (AI44231, AI38446, and U19AI083022).

Received for publication February 25, 2011, and accepted in revised form June 22, 2011.

Address correspondence to: Jeffrey N. Weiser, 402A Johnson Pavilion, University of Pennsylvania, Philadelphia, Pennsylvania, 19104-6076, USA. Phone: 215.573.3511; Fax: 215.573.4856; E-mail: weiser@mail.med.upenn.edu. 
1. Bogaert D, De Groot R, Hermans PW. Streptococcus pneumoniae colonisation: the key to pneumococcal disease. Lancet Infect Dis. 2004;4(3):144-154.

2. Vu HT, et al. Association between nasopharyngeal load of Streptococcus pneumoniae, viral coinfection, and radiologically confirmed pneumonia in Vietnamese children. Pediatr Infect Dis J. 2011;30(1):11-18.

3. Tong HH, Weiser JN, James MA, DeMaria TF. Effect of influenza A virus infection on nasopharyngeal colonization and otitis media induced by transparent or opaque phenotype variants of Streptococcus pneumoniae in the chinchilla model. Infect Immun. 2001;69(1):602-606.

4. Morens DM, Taubenberger JK, Fauci AS. Predominant role of bacterial pneumonia as a cause of death in pandemic influenza: implications for pandemic influenza preparedness. J Infect Dis. 2008; 198(7):962-970.

5. Kim PE, Musher DM, Glezen WP, Rodriguez-Barradas MC, Nahm WK, Wright CE. Association of invasive pneumococcal disease with season, atmospheric conditions, air pollution, and the isolation of respiratory viruses. Clin Infect Dis. 1996;22(1):100-106.

6. van der Sluijs KF, et al. IL-10 is an important mediator of the enhanced susceptibility to pneumococcal pneumonia after influenza infection. J Immunol. 2004;172(12):7603-7609.

7. McCullers JA, Rehg JE. Lethal synergism between influenza virus and Streptococcus pneumoniae: characterization of a mouse model and the role of platelet-activating factor receptor. J Infect Dis. 2002;186(3):341-350.

8. McCullers JA, Bartmess KC. Role of neuraminidase in lethal synergism between influenza virus and Streptococcus pneumoniae. J Infect Dis. 2003. 187(6):1000-1009.

9. Hatta M, et al. Growth of H5N1 influenza A viruses in the upper respiratory tracts of mice. PLoS Pathog. 2007;3(10):1374-1379.

10. Isaacs A, Lindenmann J. Virus interference. I. The interferon. Proc R Soc Lond B Biol Sci. 1957; 147(927):258-267.

11. Theofilopoulos AN, Baccala R, Beutler B, Kono DH. Type I interferons (alpha/beta) in immunity and autoimmunity. Annu Rev Immunol. 2005;23:307-336.

12. Tough DF, Borrow P, Sprent J. Induction of bystander T cell proliferation by viruses and type I interferon in vivo. Science. 1996;272(5270):1947-1950.

13. Matikainen $S$, et al. Influenza $A$ and sendai viruses induce differential chemokine gene expression and transcription factor activation in human macrophages. Virology. 2000;276(1):138-147.

14. Joyce EA, Popper SJ, Falkow S. Streptococcus pneumoniae nasopharyngeal colonization induces type I interferons and interferon-induced gene expression. BMC Genomics. 2009;10:404.

15. Zhang Z, Clarke TB, Weiser JN. Cellular effectors mediating Th17-dependent clearance of pneumococcal colonization in mice. J Clin Invest.
2009;119(7):1899-1909.

16. McCool TL, Weiser JN. Limited role of antibody in clearance of Streptococcus pneumoniae in a murine model of colonization. Infect Immun. 2004; 72(10):5807-5813.

17. Davis KM, Nakamura S, Weiser JN. Nod2 sensing of lysozyme-digested peptidoglycan promotes macrophage recruitment and clearance of S. pneumoniae colonization in mice. J Clin Invest. 2011:121(9):3666-3676.

18. McCool TL, Cate TR, Moy G, Weiser JN. The immune response to pneumococcal proteins during experimental human carriage. J Exp Med. 2002;195(3):359-365.

19. Diavatopoulos DA, et al. Influenza A virus facilitates Streptococcus pneumoniae transmission and disease. FASEB J. 2010;24(6):1789-1798

20. Stenfors LE, Raisanen S. Occurrence of middle ear pathogens in the nasopharynx of young individuals. A quantitative study in four age groups. Acta Otolaryngol. 1990;109(1-2):142-148.

21. Gwaltney JM Jr, Sande MA, Austrian R, Hendley JO. Spread of Streptococcus pneumoniae in families. II. Relation of transfer of S. pneumoniae to incidence of colds and serum antibody. J Infect Dis. 1975; 132(1):62-68.

22. McCullers JA, McAuley JL, Browall S, Iverson $\mathrm{AR}$, Boyd KL, Henriques Normark B. Influenza enhances susceptibility to natural acquisition of and disease due to Streptococcus pneumoniae in ferrets. J Infect Dis. 2010;202(8):1287-1295.

23. McCullers JA. Insights into the interaction between influenza virus and pneumococcus. Clin Microbiol Rev. 2006;19(3):571-582.

24. Winter C, et al. Lung-specific overexpression of CC chemokine ligand (CCL) 2 enhances the host defense to Streptococcus pneumoniae infection in mice: role of the CCL2-CCR2 axis. J Immunol. 2007; 178(9):5828-5838.

25. Zimmerer JM, Lesinski GB, Radmacher MD, Ruppert A, Carson WE 3rd. STAT1-dependent and STAT1independent gene expression in murine immune cells following stimulation with interferon-alpha. Cancer Immunol Immunother. 2007;56(11):1845-1852.

26. Shahangian A, et al. Type I IFNs mediate development of postinfluenza bacterial pneumonia in mice. J Clin Invest. 2009;119(7):1910-1920.

27. Antonelli LR, et al. Intranasal Poly-IC treatment exacerbates tuberculosis in mice through the pulmonary recruitment of a pathogen-permissive monocyte/macrophage population. J Clin Invest. 2010; 120(5):1674-1682.

28. Jia T, Leiner I, Dorothee G, Brandl K, Pamer EG. MyD88 and type I interferon receptor-mediated chemokine induction and monocyte recruitment during Listeria monocytogenes infection. J Immunol. 2009; 183(2):1271-1278.

29. Monroe KM, McWhirter SM, Vance RE. Induction of type I interferons by bacteria. Cell Microbiol.
2010;12(7):881-890.

30. Ratner AJ, Aguilar JL, Shchepetov M, Lysenko ES, Weiser JN. Nod1 mediates cytoplasmic sensing of combinations of extracellular bacteria. Cell Microbiol. 2007;9(5):1343-1351.

31. Ratner AJ, Hippe KR, Aguilar JL, Bender MH, Nelson AL, Weiser JN. Epithelial cells are sensitive detectors of bacterial pore-forming toxins. J Biol Chem. 2006;281(18):12994-12998.

32. Malley R, et al. Recognition of pneumolysin by Toll-like receptor 4 confers resistance to pneumococcal infection. Proc Natl Acad Sci U S A. 2003; 100(4):1966-1971.

33. Charrel-Dennis M, et al. TLR-independent type I interferon induction in response to an extracellular bacterial pathogen via intracellular recognition of its DNA. Cell Host Microbe. 2008;4(6):543-554.

34. Parker D, et al. Streptococcus pneumoniae DNA initiates type I interferon signaling in the respiratory tract. MBio. 2011;2(3):e00016-e0011.

35. Pandey AK, et al. NOD2, RIP2 and IRF5 play a critical role in the type I interferon response to Mycobacterium tuberculosis. PLoS Pathog. 2009;5(7):e1000500.

36. Herskovits AA, Auerbuch V, Portnoy DA. Bacterial ligands generated in a phagosome are targets of the cytosolic innate immune system. PLoS Pathog. 2007;3(3): e51

37. Weiser JN, Austrian R, Sreenivasan PK, Masure HR Phase variation in pneumococcal opacity: relationship between colonial morphology and nasopharyngeal colonization. Infect Immun. 1994;62(6):2582-2589.

38. Matthias KA, Roche AM, Standish AJ, Shchepetov M, Weiser JN. Neutrophil-toxin interactions promote antigen delivery and mucosal clearance of Streptococcus pneumoniae. J Immunol. 2008;180(9):6246-6254.

39. Muller U, et al. Functional role of type I and type II interferons in antiviral defense. Science. 1994; 264(5167):1918-1921.

40. Kim JO, Weiser JN. Association of intrastrain phase variation in quantity of capsular polysaccharide and teichoic acid with the virulence of Streptococcus pneumoniae. J Infect Dis. 1998;177(2):368-377.

41. Clarke TB, Davis KM, Lysenko ES, Zhou AY, Yu Y, Weiser JN. Recognition of peptidoglycan from the microbiota by Nod1 enhances systemic innate immunity. Nat Med. 2010;16(2):228-231.

42. McKinstry KK, et al. IL-10 deficiency unleashes an influenza-specific Th17 response and enhances survival against high-dose challenge. J Immunol. 2009;182(12):7353-7363.

43. Beisswenger C, Lysenko ES, Weiser JN. Early bacterial colonization induces toll-like receptor-dependent transforming growth factor beta signaling in the epithelium. Infect Immun. 2009;77(5):2212-2220.

44. Gautier $\mathrm{G}$, et al. A type I interferon autocrine-paracrine loop is involved in Toll-like receptor-induced interleukin-12p70 secretion by dendritic cells. J Exp Med. 2005;201(9):1435-1446. 